\title{
Estratégias aos cuidados na Atenção Primária no Sistema Único de Saúde: uma revisão integrativa
}

\author{
Strategies for care in Primary Care in the Unique Health System: a integrative review
}

Estrategias para la atención en la Atención Primaria en el Sistema Único de Salud: una revisión integradora

Jessica Morgana Lisboa de Oliveira ${ }^{1 *}$, David Breda Rêgo Machado1, Íris Régia Ventura Barros ${ }^{1}$, Ellen Marcella Freire Padilha ${ }^{1}$, Anderson de Oliveira Rocha ${ }^{1}$, Izabel Cristina Gomes de Mendonça ${ }^{1}$, Michelle Leão Bittencourt Brandão Medeiros ${ }^{1}$.

\section{RESUMO}

Objetivo: Analisar a integralidade em saúde, especialmente, observar princípios e convicções sobre a grande relevância da atenção primária durante o processo de atendimento no Sistema Único de Saúde (SUS). Métodos: Referiu-se à uma revisão de literatura, com interpelações descritivas com caráter informativo, em que os processos de formulações foram estabelecidos por meio de buscas por literaturas cientificas, em bases de dados, tendo como descritores: Atenção Primária à Saúde, Comportamentos multiprofissionais e Sistema Único de Saúde. Resultados: A atenção primária é a essencial porta de entrada sendo o ponto central na articulação da acessibilidade dos pacientes ao SUS, dos quais norteia-se através dos conceitos da acessibilidade, dos cuidados, conexões, continuação e integralidade. Para pôr em prática tais princípios, a Atenção Básica apresenta ações, atendendo as diversidades das necessidades de saúde, buscando sempre o bem-estar de todos os usuários. Considerações finais: É de suma importância a elaboração de debates que busquem a solidificação das mais diferentes formas do atendimento aos pacientes, tendo em vista que é de fundamental relevância o engajamento de toda a equipe multiprofissional, buscando sempre atender as expectativas ao que se refere à atenção primária de saúde, com a intenção de que a integralidade seja atingida.

Palavras-chave: Assistência ao paciente, Atenção Primária à Saúde, Sistema Único de Saúde.

\begin{abstract}
Objective: Analyze integrality in health, especially, observe principally, observe principles and convictions about the great relevance of primary care during the care process in the Unified Health System (SUS). Methods: It referred to a literature review, with descriptive questions with an informative character, in which the formulation processes were established through searches for scientific literature, in databases, having as descriptors: Primary Health care, Multiprofessional behaviors and System Single Health. Results: Primary care is the essential gateway and is the central point in articulating the accessibility of patients to SUS, of which it is guided through the concepts of accessibility, care, connections, continuity and integrality. In order to put these principles into practice, primary Care presents actions, meeting the diversity of health needs, always seeking the well-being of all users. Final considerations: It is of utmost importance to elaborate debates that seek the consolidation of the most different forms of patient care, bearing in mind that the engagement of the entire multidisciplinary team is of fundamental importance, always seeking to meet the expectations regarding Primary Health Care. Health, with the intention that comprehensiveness is achieved.
\end{abstract}

Keywords: Patient care, Primary Health Care, Unified Health System.

\section{RESUMEN}

Objetivo: Analice la investigación sobre la integralidad en salud, especialmente, observe los principios y convicciones sobre la gran relevancia de la atención primaria durante el proceso de atención en el Sistema Único de Salud (SUS). Métodos: Se refería a una revisión de la literatura, con preguntas descriptivas con carácter informative, en la cual los procesos de formulación se establecieron mediante búsquedas de literatura científica, en bases de datos, que tenían como descriptores: Atención primaria de salud, comportamientos y sistemas multiprofesionales salud individual. Resultados: La atención primaria es la puerta de entrada esencial y es el punto central en la accesibilidad de los pacientes a SUS, de la cual se guia a través de los

${ }^{1}$ Centro Universitário CESMAC (CESMAC), Maceió - AL. `E-mail: jjmorgana@outlook.com 
conceptos de accesibilidad, atención, conexiones, continuidad e integralidad. Para poner en práctica estos principios, la Atención Primaria presenta acciones que satisfacen la diversidad de las necesidades de salud, buscando siempre el bienestar de todos los usuarios. Consideraciones finales: Es de suma importancia elaborar debates que busquen la consolidación de las formas más diferentes de atención al paciente, teniendo en cuenta que la participación de todo el equipo multiprofesional es de fundamental importancia, siempre buscando satisfacer las expectativas con respecto a la atención primaria de salud. Salud, con la intención de que se logre la amplitud.

Palabras clave: Atención al paciente, Atención Primaria de Salud, Sistema Único de Salud.

\section{INTRODUÇÃO}

Os vigentes pontos de vistas relacionados ao processo de desenvolvimento da Atenção Primária à Saúde (APS) foi apresentada pela primeira vez no Reino Unido, no ano de 1920, nas descrições feitas por Dawson do qual recomendou uma determinada ordenação ao que se referia ao sistema de atenção à saúde em inúmeros pontos que eram cruciais, entre eles foram destacados: a assistência de forma domiciliar, os pontos de atenção à saúde primária, os centros de saúde secundários dos quais a complexidade do atendimento prestado neste nível é maior e o paciente já possui um contato direto com o profissional da saúde responsável, os serviços suplementares dos quais são observados critérios como efetividade, eficiência, equidade, acesso, centralidade no paciente e segurança e por fim porém não menos importante os hospitais de ensino (MENDES EV, 2015).

O documento proposto por Dawson demonstrou de forma bastante significativa os respectivos papeis e importância de cada grau referente ao estabelecimento da atenção e as correlações que careceriam existir diante do respectivo assunto para só assim retratar o contexto instituidor das regionalizações dos sistemas de atenção à saúde estruturada com embasamento na população daquela determinada localização, ocasionando dessa maneira inspirado a inúmeras organizações referente à essas metodologias referente os modelos de sistemas, em diversos países em uma esfera global (COSTA EK, et al., 2019).

Pode-se relevar que o modo exemplar referente à cuidados fundamentados em campanhas de saúde coletivas de forma publica dos quais eram direcionados a feitos pioneiros de forma positiva foram estabelecidos principalmente ao padrão francês do século XIX, e os países americanos que vieram a destacar-se no começo do século XX dos quais já se proporcionavam e estabeleciam concepções padronizadas, dos quais vários foram estabelecidos de acordo com os padrões da APS que foram visando maiores questões de como lidar com a saúde e enquadraram entre seus cuidados os fatores de risco de uma estimada população, planejamentos descentralizados e territoriais, assistencialismo a todos os cidadãos, medidas preventivas, educacionais e sanitárias abrangendo também ações ambientais (ARANTES LJ, et al., 2016).

No Brasil, desde o ano de 2004, determinações dos órgãos federais inicializaram a apontar a verdadeira relevância do monitoramento da qualidade e das características referente a expressão resolutiva ao que se referia a Estratégia da Saúde da Família (ESF). Averiguações de julgamento e acompanhamento com eixo fixado nas particularidades da atenção básica $(A B)$ e conselhos de opiniões da respectiva proposta de qualidade (SILVA RM, et al., 2018).

Desta maneira pode ser citado exemplos como: O Programa Nacional de Melhoria do Acesso e da Qualidade da Atenção Básica (PMAQ-AB), dos quais o seu início foi em 2012, fazendo-se componente das vigentes resoluções do Ministério da Saúde buscando estabelecer modificações nas situações de infraestrutura sabendo-se que essa parte é uma área vital para o desenvolvimento socioeconômico e desempenho das unidades básicas de saúde (UBSs), levando em consideração a sua conceituação ao que se refere a acessibilidade e o desempenho de trabalho $A B$ por intermédio de processos ininterruptos de pareceres avaliativos e estímulos financeiros (BRASIL, 2011).

O documento oficial estabelecido pela Portaria Ministerial no 2.436, de 21 de Setembro de 2017, referiuse acima de que as Políticas Nacionais de Atenções Básicas (PNAB) dos quais denota direcionamentos quanto aos processos organizacionais e dos desempenhos das entidades que envolvem à saúde, designa em seus critérios a ênfase na distribuição do constituinte relacionado ao assistencialismo da atenção básica, 
reforçando ainda que a Atenção Básica será a fundamental "porta de entrada" dos usuários nos sistemas de saúde. Ou seja, é o atendimento inicial possuindo equipes Multidisciplinares engajadas para seu maior crescimento e solidificação (BRASIL, 2017).

Torna-se indispensável um prevê estabelecimento do esclarecimento das definições sobre "Atenção Básica" e "Atenção Primária à Saúde" motivo de aprendizagem da referida pesquisa. Em função disso, Mello GA, et al. (2009) expressam que existe uma continua desentendimento em cima dessa nomenclatura adequada para mencionar o grau inicial sobre a atenção à saúde. No Brasil, o termo referente à $A B$ se oficializou através do Governo Federal, apesar de que nas declarações oficiais brasileiras, reconhece-se uma maior utilização do termo Atenção Primária à Saúde.

Essas terminologias são capazes de serem empregues como mesma expressão, desde que esses termos não venham a ser uma contrariedade especulada, dado que essas imprecisões terminológicas preconizam uma rivalidade ocasionada por causa de ideologias distintas, em discordância com a propensões internacionais da utilização de atenção primária. No entanto, ao ser comparado de modo direto os seus princípios e conceitos das inúmeras definições referentes ao primeiro nível de atenção em saúde, existindo defrontação de concepções, especialmente no que soa ao desenvolvimento histórico, conhecimentos esses que nem sempre são retratadas (BONELLO AADLM e CORRÊA CRS, 2014).

Não existe irresoluções a respeito das consequências benéficas ao que se refere à saúde da sociedade com a implantação da Atenção Primária à Saúde como sendo um componente fundamental para o decorrer da ordenação das prestações de serviços de saúde no Brasil, no entanto, por causa das dessemelhanças de condições as suas capacidades conseguem serem diminuídas causando indagações a respeito da sua confiabilidade como organizadores dos sistemas de saúde prestados (ARANTES LJ, et al., 2016).

Em razão de motivos que envolvem dimensões políticas, socioeconômicas, aspectos visionários profundos e complexos, variados, esse fator heterogêneo pode ser analisado como sendo um fator limitante para os serviços, atividades e ações relacionada à saúde. É evidente que o padrão utilizado de APS no Brasil dispõe de várias competências, por exemplo, dispondo acessos de saúde a população que ao longo da história a maioria eram excluídas e não possuíam amparos, e hoje em dia existe assistência tanto na zona rural quanto urbana, diminuindo assim as mortalidades, e a falta de instrução de muitos (SENNA CMM e SANTOS AM, 2017).

Inúmeros artigos de bases científicas demonstram a real incitações e vulnerabilidades que devem serem exceder na conjuntura referente a APS no Brasil, entre eles consegue-se apresentar os problemas de ingressão no sistema, apontando desde a entrada para as assistências aos cidadãos. Um dos fundamentais problemas se constituem nas promoções de ofertas insuficientes quando comparadas com a demanda nos serviços de saúde, fatores particulares como os aspectos demográficos, as rendas familiares, questões culturais de cada cidadão, locomoção, antecedentes de assistências recebidas (BONELLO AADLM e CORRÊA CRS, 2014; SILVA RM, et al., 2018).

Dessa maneira, o respectivo artigo possui como propósito primário reconhecer por meio da literatura científica, as reais capacidades e vulnerabilidades que ocorre no suporte de atendimento prestado aos usuário do Sistema Único de Saúde (SUS) em suas unidades básicas de saúde, demonstrando assim os fundamentais motivos que intricam o serviço prestado a saúde e acentuar a verdadeira relevância dos cuidados básicos a fim de possibilitar a completude e a relação mútua entre o usuário e o profissional de saúde dos quais são debatidos no campo da saúde.

\section{MÉTODOS}

Trata-se de uma revisão integrativa estabelecida através de uma revisão de literatura com abordagem descritiva e caráter informativo realizada entre o período de janeiro de 2020 a junho de 2020, na qual artigos da Literatura Internacional em Ciência da Saúde (MEDLINE), da Literatura Latino Americana e do Caribe em Ciências da Saúde (LILACS), e da Scientific Electronic Library Online (SCIELO), além de livros à disposição no acervo bibliográfico da biblioteca central do Centro Universitário CESMAC (Centro de Ensino Superior de Maceió) foram analisados. 
Para isso o embasamento do respectivo estudo foi determinado através dos seguintes critérios: Foram consideradas teses, dissertações, monografias, publicações do Ministério da Saúde e Organizações Internacionais, no idioma inglês e português, escolhidos os trabalhos publicados nos últimos 7 anos (2014 a 2020).

De acordo com a forma metodológica descrita por Silva Neto JM, et al., (2019), os artigos foram provindos através de 5 pesquisadores: no começo estabeleceu-se o tema a ser estudado e posteriormente, foram feitas as devidas leituras de forma aprofundada dos respectivos resumos para o aproveitamento dos processos descritivos, tornando-os assim mais restritos.

Após selecionados os artigos, foram organizados de acordo com a extensão das amostragens, o objetivo, a metodologia, as normas esclarecidas e seus resultados. As restrições adotadas foram os períodos de publicações e artigos de relatos de casos. Os principais pontos explanados nos trabalhos envolviam as políticas de humanização durante o atendimento ao paciente e seu acolhimento e o funcionamento do SUS. Os descritores utilizados foram: Avaliação de Serviços de Saúde, Atenção Primária à Saúde, Estratégia Saúde da Família, Atenção Primária à Saúde.

\section{RESULTADOS E DISCUSSÃO}

Foram acordados 36 artigos que se certificavam da integridade e totalidade nas bases de dados citadas. Ao decorrer do processo evolutivo da pesquisa, 16 possuíam duplicidade nas mencionadas fontes de pesquisa, mantendo-se 20 artigos para o processo qualificativo do referente material, no entanto quatro foram excluídos por não disporem de maneira propicia as diretrizes fundamentais da construção do estudo. Em consequência 16 artigos foram apurados para a avaliação por intermédio das sínteses presente, quatro foram excluídos, desta forma, foram definidos 19 artigos para o deslindamento e constituição imprescindíveis para o progresso do estudo.

Depois do estabelecimento das conjunturas do respectivo estudo, não foi posta outras fontes de pesquisa ao concluir as verificações informativas que se propuseram a respectiva pesquisa, a revisão foi estabelecida por 19 artigos científicos de conceituada relevância, e o fluxograma demostra as formas de análises que foram produzidas mediante de apuramento realizado por pesquisas estipuladas para a validação direta desta análise literária (Figura 1).

Figura 1 - Fluxograma dos estudos identificados.

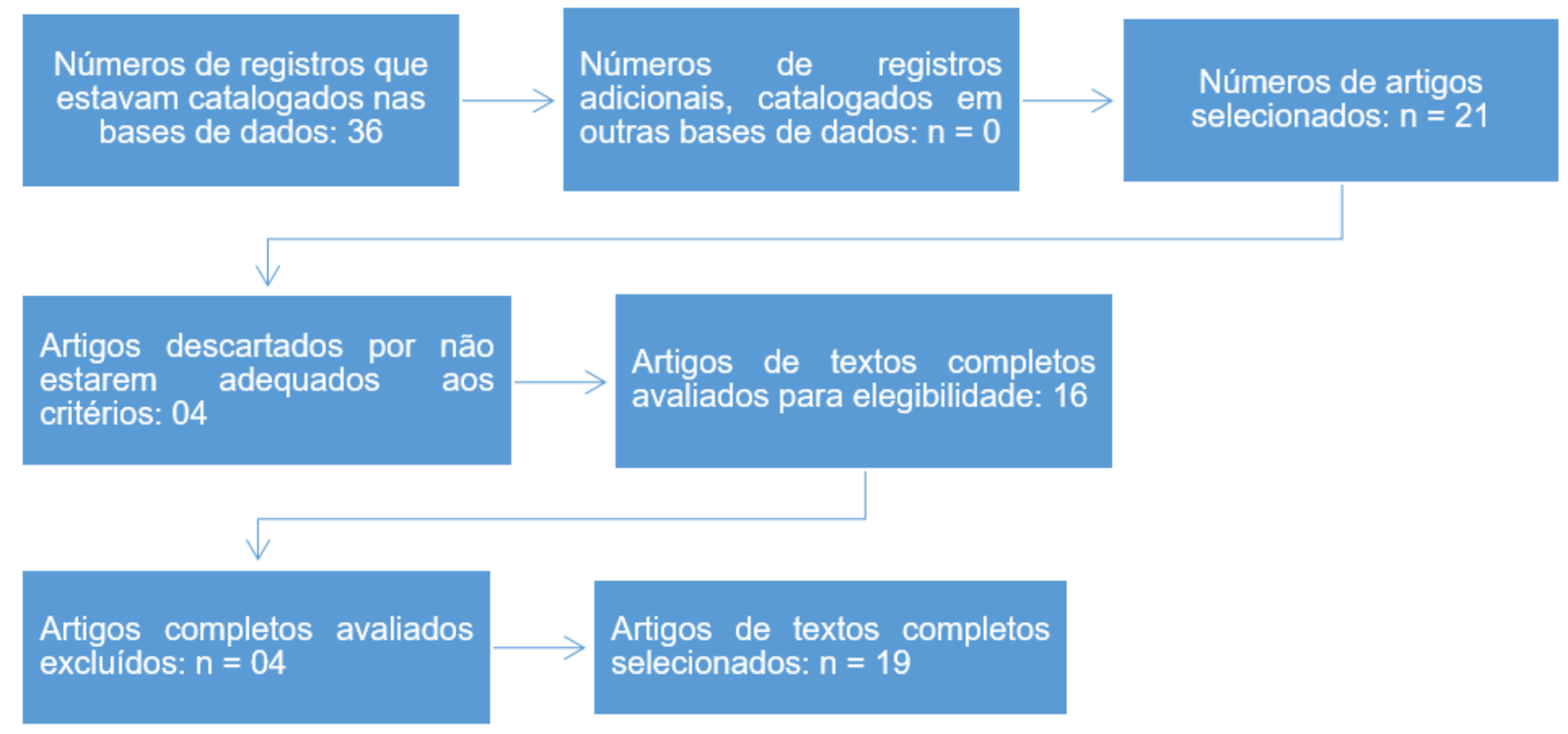

Fonte: Oliveira JML et al., 2021.

Os artigos possuíam suas informações através dos tópicos: artigo/autor, ano, periódico, objetivo e resultados (Quadro 1). 
Quadro 1-Delineamento, métodos e principais desfechos dos estudos selecionados.

\begin{tabular}{|c|c|c|c|c|}
\hline Artigo & Autor/Ano & Periódico & Objetivo & Resultados \\
\hline $\begin{array}{l}\text { Avaliação de serviços de saúde } \\
\text { bucal: um estudo comparativo. }\end{array}$ & $\begin{array}{l}\text { BRUNHAUSER AL, } \\
\text { et al., } 2013 .\end{array}$ & RFO PASSO FUNDO & $\begin{array}{l}\text { Avaliar o acesso e a satisfação dos usuários dos } \\
\text { serviços odontológicos do SUS, comparando } \\
\text { usuários da ESF com usuários de UBS } \\
\text { convencionais, em Não-Me-Toque/RS, Brasil. }\end{array}$ & $\begin{array}{l}\text { Testes de qui-quadrado demonstraram que } 93 \% \text { dos } \\
\text { usuários da ESF apresentaram-se muito/totalmente } \\
\text { satisfeitos e apenas } 7 \% \text { mostraram-se satisfeitos. Já no } \\
\text { grupo de usuários das UBSs convencionais, } 86 \% \\
\text { apresentaram-se muito/ totalmente satisfeitos e outros } \\
14 \% \text { mostraram-se satisfeitos. }\end{array}$ \\
\hline $\begin{array}{l}\text { Aposição da estratégia saúde da } \\
\text { família na rede de atenção à } \\
\text { saúde na perspectiva das } \\
\text { equipes e usuários participantes } \\
\text { do programa nacional para } \\
\text { melhoria do acesso e da } \\
\text { qualidade da atenção básica. }\end{array}$ & $\begin{array}{l}\text { FAUSTO MCR, et } \\
\text { al.,2014. }\end{array}$ & Saúde Debate & $\begin{array}{l}\text { Analisar a posição da Estratégia Saúde da } \\
\text { Família na rede de atenção à saúde sob a } \\
\text { perspectiva das equipes de Saúde da Família e } \\
\text { dos usuários participantes do Programa Nacional } \\
\text { para Melhoria do Acesso e da Qualidade da } \\
\text { Atenção Básica. }\end{array}$ & $\begin{array}{l}\text { Os resultados indicam que as equipes atuam cada vez } \\
\text { mais como porta de entrada preferencial, atendendo a } \\
\text { demandas diversas e exercendo a função de filtro para } \\
\text { a atenção especializada. Contudo, persistem } \\
\text { importantes barreiras organizacionais para acesso, os } \\
\text { fluxos estão pouco ordenados, a integração da APS à } \\
\text { rede ainda é incipiente e inexiste coordenação entre } \\
\text { APS e atenção especializada. }\end{array}$ \\
\hline $\begin{array}{l}\text { A qualidade da atenção primária } \\
\text { no programa mais médicos- a } \\
\text { experiência dos médicos e } \\
\text { usuários. }\end{array}$ & $\begin{array}{l}\text { ORGANIZAÇÃO } \\
\text { PAN-AMERICANA } \\
\text { DA SAÚDE, } 2017 .\end{array}$ & $\begin{array}{l}\text { Biblioteca Virtual da } \\
\text { Saúde (BVS) }\end{array}$ & $\begin{array}{l}\text { Avaliar a força da APS à saúde nas equipes das } \\
\text { estratégias saúde da família de todo Brasil, por } \\
\text { meio da experiência de usuários dos serviços e } \\
\text { de médicos cubanos participantes do programa } \\
\text { mais médicos. }\end{array}$ & $\begin{array}{l}\text { As estratégias nacionais para ampliação e qualificação } \\
\text { da APS no Brasil têm feito escores mais altos na maioria } \\
\text { das regiões, apesar da dificuldade de acesso às } \\
\text { Unidades de Saúde da Família, aqueles pacientes que } \\
\text { conseguem acessá-las desenvolvem relações de } \\
\text { confiança com os médicos, como demonstram os } \\
\text { Escores de Longitudinalidade. }\end{array}$ \\
\hline $\begin{array}{l}\text { Estratégias para consolidação } \\
\text { da coordenação do cuidado pela } \\
\text { atenção básica. }\end{array}$ & $\begin{array}{l}\text { ALMEIDA PF, et al., } \\
2017 .\end{array}$ & $\begin{array}{l}\text { Scientific Electronic } \\
\text { Library (SCIELO) }\end{array}$ & $\begin{array}{l}\text { Avaliar a organização da porta de entrada, } \\
\text { resolutividade e continuidade do cuidado, } \\
\text { integração horizontal, organização dos fluxos e } \\
\text { acesso à rede de referência, continuidade } \\
\text { informacional e comunicação entre profissionais }\end{array}$ & $\begin{array}{l}\text { Os resultados indicaram que a atenção primária em } \\
\text { saúde se consolidou como porta de entrada preferencial. }\end{array}$ \\
\hline $\begin{array}{l}\text { Construção e validação de um } \\
\text { instrumento para avaliação dos } \\
\text { serviços públicos de saúde bucal } \\
\text { na atenção primária à saúde sob } \\
\text { a ótica dos usuários } \\
\text { (ASBAPusuário). }\end{array}$ & SANCHEZ HF, 2013. & $\begin{array}{l}\text { Biblioteca Digital } \\
\text { UFMG }\end{array}$ & $\begin{array}{l}\text { Avaliar um instrumento, na forma de } \\
\text { questionário, capaz de avaliar a saúde bucal na } \\
\text { atenção primária à saúde sob a ótica de usuários. }\end{array}$ & $\begin{array}{l}\text { A utilização do questionário por parte dos usuários } \\
\text { poderá representar um estímulo à participação cidadã } \\
\text { nos rumos dos serviços de saúde e os resultados } \\
\text { obtidos na aplicação do questionário poderão } \\
\text { sensibilizar gestores a qualificar os serviços de saúde e } \\
\text { as práticas profissionais e, em última análise, tornar } \\
\text { realidade a construção de um sistema público de saúde } \\
\text { à altura de sua existência, que são os próprios usuários. }\end{array}$ \\
\hline $\begin{array}{l}\text { Avaliação da atenção primária: } 0 \\
\text { ponto de vista de usuários. }\end{array}$ & $\begin{array}{l}\text { GONTIJO TL, et al., } \\
2017 .\end{array}$ & Saúde Debate & $\begin{array}{l}\text { Avaliar atributos da Atenção Primária à Saúde } \\
\text { (APS), sob a percepção de usuários. }\end{array}$ & $\begin{array}{l}\text { Conclui-se que os atributos da APS analisados } \\
\text { receberam avaliação heterogênea e a maioria necessita } \\
\text { de um olhar mais atento e investimento por parte dos } \\
\text { gestores e profissionais. }\end{array}$ \\
\hline
\end{tabular}




\begin{tabular}{|c|c|c|c|c|}
\hline Artigo & Autor/Ano & Periódico & Objetivo & Resultados \\
\hline $\begin{array}{l}\text { Percepções de usuários } \\
\text { atendidos em um Pronto } \\
\text { Atendimento: olhar sobre a } \\
\text { Atenção Primária à Saúde. }\end{array}$ & $\begin{array}{l}\text { BARATIERI T, et al., } \\
2017 .\end{array}$ & $\begin{array}{l}\text { Revista de Saúde } \\
\text { Pública do Paraná. }\end{array}$ & $\begin{array}{l}\text { Compreender os motivos que levam os usuários } \\
\text { a buscar as Unidades de Pronto Atendimento } \\
\text { (UPA) quando o problema de saúde poderia ser } \\
\text { resolvido no âmbito da Atenção Primária à Saúde } \\
\text { (APS). }\end{array}$ & $\begin{array}{l}\text { Descompasso entre a visão médico-centrada e a } \\
\text { inadequação das equipes de saúde da APS para } \\
\text { atenção à demanda espontânea; acolhimento deficitário } \\
\text { na APS gera dificuldades de acesso e baixa } \\
\text { resolutividade e desarticulação entre UPA e APS. } \\
\text { Necessidade de estruturação da coordenação do } \\
\text { cuidado. Conclui-se que as UPAs são vistas pela } \\
\text { população como mais resolutivas, havendo necessidade } \\
\text { de ordenar fluxos de atendimento e educar/informar a } \\
\text { população sobre seu funcionamento. }\end{array}$ \\
\hline $\begin{array}{l}\text { Análise da eficiência na atenção } \\
\text { primária à saúde sob a ótica dos } \\
\text { profissionais da área. }\end{array}$ & $\begin{array}{l}\text { CABRAL KFD, et al., } \\
2016 .\end{array}$ & $\begin{array}{l}\text { Revista de Gestão em } \\
\text { Sistemas de Saúde }\end{array}$ & $\begin{array}{l}\text { Analisar a eficiência da Atenção Primária à } \\
\text { Saúde, avaliando as experiências de municípios } \\
\text { brasileiros do estado de Minas Gerais no que se } \\
\text { refere à sua eficiência na promoção da saúde. }\end{array}$ & $\begin{array}{l}\text { Observou-se que as dificuldades apontadas em comum } \\
\text { nos municípios analisados se referem à falta de recursos } \\
\text { ou sua limitação, bem como às condições educacionais } \\
\text { da população e sua baixa participação e conhecimento } \\
\text { sobre a Estratégia Saúde da Família. Conclui-se que há } \\
\text { grande necessidade de trabalhos intersetoriais que } \\
\text { apoiem ações de promoção da saúde já existentes, bem } \\
\text { como novas estratégias que podem elevar o nível de } \\
\text { conhecimento da população, dos profissionais e } \\
\text { também dos gestores como forma de se melhorar o } \\
\text { desempenho da promoção da saúde. }\end{array}$ \\
\hline $\begin{array}{l}\text { Atenção Primária à Saúde na } \\
\text { Agenda Pública Brasileira: } \\
\text { dilemas entre focalização e } \\
\text { universalidade. }\end{array}$ & $\begin{array}{l}\text { SENNA CMM e } \\
\text { SANTOS AM, } 2017 .\end{array}$ & $\begin{array}{l}\text { Revista de Políticas } \\
\text { Públicas }\end{array}$ & $\begin{array}{l}\text { Reflexão sobre os desafios postos à Atenção } \\
\text { Primária à Saúde no contexto brasileiro. }\end{array}$ & $\begin{array}{l}\text { Houve um esforço importante de expansão da oferta de } \\
\text { serviços de saúde da APS nas últimas décadas, mas, } \\
\text { observa-se que essa expansão se deu de forma } \\
\text { desigual e heterogênea no território nacional. As } \\
\text { recentes iniciativas de constituição das Redes de } \\
\text { Atenção à Saúde confirmam o ainda frágil desempenho } \\
\text { da APS como eixo organizador da rede de serviços de } \\
\text { saúde e política universal. Tal reconhecimento exige } \\
\text { recuperar uma perspectiva crítica de análise sobre os } \\
\text { constrangimentos que se impõem ao SUS. }\end{array}$ \\
\hline $\begin{array}{l}\text { Acesso às consultas médicas } \\
\text { nos serviços públicos de saúde. }\end{array}$ & $\begin{array}{l}\text { DIAS OV, et al., } \\
2016 .\end{array}$ & $\begin{array}{l}\text { Revista Brasileira de } \\
\text { Medicina de Família e } \\
\text { Comunidade. }\end{array}$ & $\begin{array}{l}\text { Avaliar o acesso às consultas médicas em } \\
\text { serviços públicos de saúde. }\end{array}$ & $\begin{array}{l}19,4 \% \text { afirmaram ter procurado um profissional/serviço } \\
\text { público de saúde nos } 15 \text { dias anteriores à entrevista. } \\
75,6 \% \text { foram na APS. A maior procura foi por parte do } \\
\text { sexo feminino e os sem plano de saúde. A maioria foi } \\
\text { atendida na primeira vez que procurou o serviço de } \\
\text { saúde pública. Pode-se destacar uma maior prevalência } \\
\text { ao não acesso quando a procura foi na APS e para } \\
\text { tratamento/reabilitação. Utilização de consultas médicas } \\
\text { no ano foi observada para } 54,4 \% \text {, com maiores médias } \\
\text { para mulheres, adultos. }\end{array}$ \\
\hline
\end{tabular}

Fonte: Oliveira JML et al., 2021.

REAS | Vol.13(7) | DOI: https://doi.org/10.25248/REAS.e7971.2021 Página 6 de 9 
O maior demarcação do momento histórico que envolveu a APS ocorreu, entretanto, no final da década de 70, onde ocorreu a primeira Conferição Internacional dos quais abrangiam os assuntos relacionados as medidas que deveriam serem tomadas a respeito aos cuidados primários que deveriam serem implantados, dos quais, até o momento era apenas ilustrada como 'Alma-Ata', nome esse representado por causa da cidade onde localiza-se no Cazaquistão das quais foi o local a realização do evento (FAUSTO MCR, et al., 2014).

Nesse contexto revolucionário 134 países de todos os continentes entraram em um denominador comum a respeito da temática e firmaram uma carta dos quais se pactuavam a ideia de transformar seus programas de saúde de forma mais abrangida e clara, onde foram considerados uma associação de princípios e valores que amplificavam de grande forma o verdadeiro significado e conceito referente à saúde, estabelecendo-se no socioeconômico (ALMEIDA PF, et al., 2017).

Em concordância com a Declaração estabelecida, o sistema de cuidados referentes à Atenção Primária no documento estabelecido na conferência foi referido como "cuidados primários" dos quais esses cuidados deveriam fazer inclusão, ao menos ao sistema educacional como dever para todos, tendo cuidados as questões referentes aos predomínios da saúde e uma obtenção aos processos metodológicos para as precauções, administração, controle, tendo como enfoque os cuidados nutricionais dos usuários que realmente necessita (DIAS OV, et al., 2016).

Sendo levado em consideração os cuidados de forma correta ao abastecimento de água de qualidade satisfatória, atenção no saneamento básico, analise ao aspecto da qualidade de vida materno-infantil, até mesmo ao organização familiar, vacinas disponíveis ao que se refere a imunização contra a maioria das doenças infecciosas existentes, e que possam a vim colocar em risco determinada população, precaução e retenções de doenças geograficamente endêmicas, buscando sempre o recursos terapêuticos adequados de doenças e provimentos de medicamentos fundamentais (BARATIERI T, et al.,2017).

Mesmo sabendo que as propostas lançadas na Alma-Ata não disponham do que realmente almejaram quando foram planejadas, a idealização de Atenção Primária submetida no referido momento influenciou as transformações referentes aos aspectos da saúde sanitária que sucedeu em vários países do mundo nas décadas de 80 e 90, até mesmo no Brasil (FAUSTO MCR, et al., 2014; SILVA RM, et al., 2018).

As adversidades ocasionadas ocorreram devido o desenvolvimento do sistema do neoliberalismo dos quais combatem, especificamente, as políticas do Estado e do Bem-Estar social, um dos preceitos básicos da social democracia, com isso a idealização foi conveniente para alguns países sendo assim transformadas e acatadas (NEVES M, et al., 2013).

Diversos países e órgãos internacionais, como foi o caso do Banco Mundial, abraçaram a APS em uma concepção focada, compreendendo a concepção da atenção primária como sendo um engajamento de atos e ações referente à saúde de pouca dificuldade, adaptada para as pessoas que dispõem de baixa renda econômica, no âmbito de diminuir a restrição socioeconômica resultante da amplificação da revolução ocasionada através do capitalismo global, afastando-se do feitio da universalista e igualdade que representava a Declaração de Alma-Ata e do conceito de defendimento da saúde como um direito de todos os cidadãos (ALMEIDA PF, et al., 2017).

Contudo, observasse que a APS na atualidade é uma das principais formas de entrada dos pacientes ao SUS e às Redes de Atenção à Saúde, sendo desta forma um local onde se concentra processos de articulações para o melhor atendimento. $\mathrm{O}$ assistencialismo primário busca de forma direta direcionar e conduzir os pacientes respeitando as condutas que envolvem os conceitos do acesso, formas coordenadas do cuidado ao usuário, estabelecimento de vínculo, constância e integralidade dos pacientes (ALMEIDA PF, et al., 2017). Visando estabelecer esse princípio, a Atenção Básica estabelece programas, atuações e condutas, levando sempre em consideração os aspectos sociais, culturais e econômicos, sendo assim imparcial nas divergentes necessidades de cada indivíduo que busca o Sistema de saúde (SENNA CMM e SANTOS AM, 2017). 
As APS, das quais pode-se afirmar é a porta de acesso com estrutura física dos quais englobam a Atenção Básica, ficam situadas nas proximidades da vida cotidiana dos respectivos usuários de determinada comunidade, representando uma conduta centralizada na proteção do ingresso a uma saúde de qualidades peculiares (BRUNHAUSER AL, et al., 2013).

As unidades fornecem diversas formas de atendimento e prestações de serviços efetivado pelo Sistema Único de Saúde, envolvendo desde o acolhimento com especificações de perigos, tratamentos terapêuticos com equipe técnica de enfermagem, médicas e Cirurgiões Dentistas para o cuidado da saúde oral, possuindo também compartilhando medicamentos prescritos e administrando de forma correta em cada paciente, além das campanhas educativas que são realizadas e vacinações, visitas domiciliares a pacientes que não possui condições físicas ou psicológicas de ir até o local do atendimento, à APS, é composta por uma equipe interdisciplinar e multiprofissional dos quais estão inclusos os desde os agentes comunitários de saúde aos enfermeiro, técnico de enfermagem, médico, Dentistas, auxiliar em saúde bucal (DIAS OV, et al., 2016).

Os aspectos que englobam os processos de palpabilidade dos casos que envolvem às políticas relacionadas a saúde tornar-se-á estabelecida com a reiteração na alto afirmação, através de exemplos no sistema público de saúde relativamente elevado ao modelo liberal do qual abrange uma filosofia política e moral baseada na liberdade, com a autorização dos representantes do Estado e buscando sempre a igualdade diante da lei, com isso observa-se que apenas as lutas continuas das ações coletivas pelo direito à saúde pode certificar um melhor atendimento (ALMEIDA PF, et al., 2017).

Pesquisas cientificas demonstram que os funcionários da saúde agem progressivamente à cada dia sendo à APS uma das principais porta de entrada para a escolha dos usuários, tendo em vista que atende a várias solicitações de demandas e desempenhando a funcionalidades através de processos de filtragem para só assim ocorrer o encaminhamento ao centro de atenção especializada. Apesar disso, existem consideráveis bloqueios institucionais e organizacionais para o respectivo atendimento, um dos pontos que podem serem levados em consideração a respeito desse empasse são referentes a falta de ordenações dos fluxos, a incorporação inclusiva da APS às redes mais especializadas continuam sendo incipiente e existe uma falta de coordenação especializada para fazer a interligação entre à Atenção Primária à Saúde e os centros de atenções especializadas (FAUSTO MCR, et al., 2014).

Com o intuito de analisar a qualidade do impulsionamento da APS na saúde dos dirigentes das estratégias de saúde da família em todo país, por meio de metodologias e técnicas estabelecidas em padrões nacionais para amplificação e conceituação da APS, foram demonstrados através de evidenciações que no Brasil tais técnicas incrementais têm potencialidades e contagens mais elevadas na grande maioria das regiões, ainda que exista adversidades nos usuários ingressos às Unidades de Saúde da Família, porém é notável que os pacientes que adquirem o estabelecimento da efetiva prestação de serviços conseguem estabelecer uma relação de confiança com os profissionais de saúde capacitados (OPAS, 2018; COSTA EK, et al., 2019).

Na ótica estabelecida por Senna CMM e Santos AM (2017), o atendimento a receptividade, ao se amplificar o processo de acessibilidade dos usuários do Sistema Único de Saúde na atenção primária, do qual se associa a assiduidade e o acompanhamento dos profissionais de saúde qualificados para uma forma de ouvir de forma ativa e definida às suas buscas, concede desta forma consultar e examinar os pacientes respeitando as diretrizes que o processo de atendimento humanizado necessita.

A metodologia das quais ocorrem as avaliações referentes à satisfação do paciente/usuário é bastante aprofundada dos quais possuem como obrigações decorrer por meio de propostas propicias para esse objetivo final. Um dos dispositivos que são qualificado para essa medição de satisfação necessitam serem fundamentados no entendimento do paciente quando exteriorizam suas opiniões a respeito da natureza dos serviços ao qual foi submetido ou recebeu. As expectações dos pacientes são particularizadas através do contexto do compromisso do profissional, das experiências vivenciadas em atendimentos anteriores e do grau de instrução do paciente (BRUNHAUSER AL, et al., 2013; NEVES M, et al., 2013). 


\section{CONSIDERAÇÕES FINAIS}

No Brasil, a ordenação da porta de entrada dos usuários é um dos componentes fundamentais ao que se refere a intensificação que a APS possui. Vários indicadores demonstram a importância desse sistema integrado, dos quais pode-se destacar: o acolhimento ao paciente, a forma e conduta de atendimento à demanda estabelecida de forma natural e a assistência de urgência e emergência. É de suma importância a elaboração de debates que busquem a solidificação das mais diferentes formas do atendimento aos pacientes, tendo em vista que é de fundamental relevância o engajamento de toda a equipe multiprofissional, buscando sempre atender as expectativas ao que se refere à atenção primária de saúde, com a intenção de que a integralidade seja atingida ao alcance de todos.

\section{REFERÊNCIAS}

1. ALMEIDA PF, et al. Estratégias para Consolidação da Coordenação do Cuidado pela Atenção Básica. Trabalho, Educação, Saúde, 2017; 15(2): 373-398.

2. ARANTES LJ, et al. Contribuições e desafios da Estratégia Saúde da Família na Atenção Primária à Saúde no Brasil: revisão da literatura. Ciência e Saúde Coletiva, 2016; 21(5): 1499-1509.

3. BARATIERI T, et al. Percepções de usuários atendidos em um Pronto Atendimento: olhar sobre a Atenção Primária à Saúde. Espaç. Saúde (Online), 2017; 18: 01.

4. BONELLO AADLM, CORREAA CRS. Acesso aos serviços básicos de saúde e fatores associados: estudo de base populacional. Revista Ciência \& Saúde Coletiva, 2014; 19(11): 4397-4406.

5. BRASIL. Manual do Ministérios de Saúde. Atenção Primária Brasília: CONASS, 2011. Disponível em: https://www.conass.org.br/bibliotecav3/pdfs/colecao2011/livro_3.pdf. Acessado em: 23 de julho de 2021.

6. BRASIL. Ministério da Saúde. PORTARIA № 2.436, DE 21 DE SETEMBRO DE 2017. Brasília-DF, 2017. Disponível em: https://bvsms.saude.gov.br/bvs/saudelegis/gm/2017/prt2436_22_09_2017.html. Acessado em: 23 de julho de 2021.

7. BRUNHAUSER AL, et al. Avaliação de serviços de saúde bucal: um estudo comparativo. Revista da faculdade de odontologia Universidade de Passo Fundo, 2013; 18(1): 24-31.

8. CABRAL KFD, et al. Análise da Eficiência na Atenção Primária à Saúde sob a Ótica dos Profissionais da Área. Revista de Gestão em Sistemas de Saúde, 2016; 5(2): 71-83.

9. COSTA EK, et al. Análise do efeito da radiofrequência no tratamento de flacidez cutânea relacionada ao processo de envelhecimento: revisão integrativa. Revista Eletrônica Acervo Saúde, 2019; Sup.31(e856): 3-4.

10. DIAS OV, et al. Acesso às consultas médicas nos serviços públicos de saúde. Revista Brasileira de Medicina de Família e Comunidade, 2016; 11(38): 1-13.

11. FAUSTO MCR, et al. A posição da Estratégia Saúde da Família na rede de atenção à saúde na perspectiva das equipes e usuários participantes do PMAQ-AB. Saúde Debate, 2014; 38: 13-33.

12. GONTIJO TL, et al. Avaliação da atenção primária: o ponto de vista de usuários. Saúde Debate, 2017; 41(114): 741 752.

13. MELLO GA, et al. Atenção Básica e Atenção Primária á Saúde - Origens e Diferenças Conceituais. Artigo de Revisão. Revista Atenção Primária à Saúde, 2009; 12(2): 204-213.

14. MENDES EV. A Construção Social da Atenção Primária À Saúde. Brasília: Conselho Nacional de Secretários de Saúde - CONASS, 2015.

15. NEVES M, et al. Atenção primária à saúde bucal no Brasil: processo de trabalho das equipes de saúde bucal. Cien Saude Colet., 2017; 24(5):1809-1820.

16. ORGANIZAÇÃO PAN-AMERICANA DA SAÚDE, OPAS. Qualidade da atenção primária no Programa Mais Médicos. A experiência dos médicos e usuários. Brasília, D.F.: OPAS; 2018. Disponível em: https://iris.paho.org/bitstream/handle/10665.2/49133/OPASBRA18029-por. pdf?sequence=1\&isAllow ed=y. Acessado em: 23 de julho de 2021.

17. SANCHEZ HF. Construção e validação de um instrumento para avaliação dos serviços públicos de saúde bucal na atenção primária à saúde sob a ótica dos usuários (ASBAP-usuário). 2013.

18. SENNA CMM, SANTOS AM. Atenção Primária à Saúde Na Agenda Pública Brasileira: Dilemas Entre Focalização e Universalidade. Revista de Políticas Públicas, 2017; 21(1): 403-424.

19. SILVA NETO JM, et al. Atenção da equipe de saúde bucal no atendimento domiciliar: Revisão integrativa. Revista Eletrônica Acervo Saúde, 2019; 37(e1958): 1-8.

20. SILVA RM, et al. Atuação da equipe de saúde bucal na atenção domiciliar na estratégia saúde da família: uma revisão integrativa. Rev. Ciência \& saúde, 2018; 26(6). 